\title{
Retrospective Study of the Risk Factors and Prevalence of Regurgitation in Dogs Undergoing General Anaesthesia
}

\author{
C. De Miguel García, G.L. Pinchbeck, A. Dugdale and J.M. Senior ${ }^{*}$
}

School of Veterinary Science, University of Liverpool, Leahurst Campus, Neston, South Wirral, CH64 7TE, UK

\begin{abstract}
The records of 5736 general anaesthetics were reviewed to estimate the prevalence of, and identify the risk factors for, the development of regurgitation during anaesthesia in dogs. Regurgitation was defined as the observed passive passage of gastric contents into the oropharynx. Several variables were evaluated using univariable and multivariable logistic regression analysis: breed, body mass, age, sex, type of procedure, expertise of anaesthetist, ASA status, drugs administered, length of surgery and anaesthesia and local techniques performed.

Results showed that larger dogs, dogs with ASA status of 3 or higher, dogs undergoing abdominal surgery, imaging procedures or both, longer anaesthetic duration, and dogs receiving medetomidine in comparison to acepromazine and an opioid were more likely to suffer an episode of regurgitation.
\end{abstract}

Keywords: Dogs, general anaesthesia, morbidity, regurgitation, risk factors.

\section{INTRODUCTION}

Gastro-oesophageal reflux (GOR), as opposed to regurgitation, involves the "silent" passage of gastric contents into only the oesophagus, and can lead to oesophageal mucosal injury [1]. In clinical studies in people, the measurement of distal oesophageal $\mathrm{pH}$ is used as the reference standard for diagnosis of the condition [2]. An episode of GOR is recorded when lower oesophageal $\mathrm{pH}$ is $<4$ or $>7.5$ by placing a $\mathrm{pH}$ measuring probe in the distal few centimetres of the oesophagus $[3,4]$.

Regurgitation has been defined as the "visible" passage of gastric contents into the oropharynx [5]. Regurgitation is therefore the visible marker of GOR and is associated with a greater likelihood of subsequent oesophageal morbidity such as oesophagitis, stricture formation, chronic cough and aspiration pneumonia $[6,7]$.

GOR is usually clinically silent, and has been reported in up to $41 \%$ of dogs undergoing abdominal surgery and from $16 \%$ to $50 \%$ of dogs undergoing anaesthesia with or without surgery $[3,8,9]$. In three different study populations of anaesthetised dogs, visible regurgitation was reported to occur in $0.42 \%-16.3 \%[3,8,10,11]$. In people, regurgitation has been described in $7.8 \%$ of cases [12].

On most occasions, GOR occurs shortly after induction of anaesthesia: $46.8 \%$ of the episodes occur within 15 minutes and $66 \%$ within 20 minutes after induction [8]. The prolongation of anaesthesia has been associated with either an increaseor no change in the incidence of GOR [7, 8] and no change in the incidence of regurgitation [11].

*Address correspondence to this author at the School of Veterinary Science, University of Liverpool, Leahurst Campus, Neston, South Wirral, CH64 7TE, UK; Tel: 0151794 6041; E-mail: J.M.Senior@liverpool.ac.uk
Risk factors for GOR in dogs and cats have been identified and include anatomical factors $[8,9,13,14]$, pharmacological factors $[3,10,15-20]$, pain and increased sympathetic tone [13], surgical factors $[1,8,9,20]$ and obstetric factors $[13,21]$.

The aims of this study were to identify the prevalence of regurgitation within dogs undergoing general anaesthesia in our hospital population and to assess possible associated risk factors.

\section{MATERIALS AND METHODS}

\section{Study Design}

A retrospective study was designed to estimate the prevalence of, and identify the risk factors for, regurgitation during general anaesthesia in dogs anaesthetised in the Small Animal Teaching Hospital, University of Liverpool.

\section{Identification of Cases and Controls}

Records of all dogs undergoing general anaesthesia between January 2008 and December 2010 were reviewed. Since 2007, details of all anaesthetics were inputted into an electronic database. Continuous variables collected were: age of patient, body mass and length of surgery and anaesthesia. Discrete variables were categorized as indicated in Table $\mathbf{1}$. Since 2008, it has been hospital policy to record an episode of intra-anaesthetic regurgitation in $\operatorname{dogs}$ and ensure appropriate treatment to these patients.

\section{Statistical Analysis}

All collected information was entered into a spreadsheet (Microsoft Excel 2007, Microsoft, Redmond, WA, USA) and the dataset was reviewed and checked for coding of all variables. Descriptive statistics were calculated for each variable.

Associations between each variable and the presence of regurgitation were assessed using univariable binomial 
Table 1. Summary of Categorical Data Collected for Each Case

\begin{tabular}{|c|c|}
\hline $\operatorname{Sex}$ & Female, Male, Female Neutered, Male neutered \\
\hline Breed (British Kennel Club Classification) & $\begin{array}{ll}- & \text { Gundogs } \\
- & \text { Herding } \\
\text { - } & \text { Hounds } \\
\text { - } & \text { Terriers } \\
\text { - } & \text { Toy breeds } \\
\text { - } & \text { Utility breeds } \\
- & \text { Working breeds } \\
- & \text { Cross breeds }\end{array}$ \\
\hline Anaesthetist & $\begin{array}{ll}- & \text { Senior anaesthetists } \\
\text { - } & \text { Anaesthesia interns } \\
\text { - } & \text { Locum/visitors } \\
\text { - } & \text { Nurses } \\
\text { - } & \text { Other veterinary members of staff not belonging to the anaesthesia department, } \\
\text { involvement of a student was also noted }\end{array}$ \\
\hline Type of procedure & $\begin{array}{ll} & \text { Gastrointestinal procedures } \\
\text { - } & \text { Non-gastrointestinal procedures } \\
\text { - } & \text { Diagnostic imaging procedures } \\
\text { - } & \text { Gastrointestinal procedures + Diagnostic imaging } \\
\text { - } & \text { Non-gastrointestinal procedures + Diagnostic imaging }\end{array}$ \\
\hline Premedication & $\begin{array}{ll}- & \text { Acepromazine } \\
- & \text { Acepromazine }+ \text { Opioid } \\
\text { - } & \text { Opioid } \\
\text { - } & \text { Medetomidine } \\
\text { - } & \text { Medetomidine }+ \text { Opioid } \\
\text { - } & \text { Miscellaneous (ketamine, benzodiazepines and combinations) }\end{array}$ \\
\hline Induction agent & $\begin{array}{ll}\text { - } & \text { Injectable anaesthetic agent } \\
\text { - } & \text { Volatile anaesthetic agent }\end{array}$ \\
\hline ASA status & $\begin{array}{ll}- & A S A 1 \\
- & A S A 2 \\
- & A S A \geq 3\end{array}$ \\
\hline Maintenance agents & $\begin{array}{ll}- & \text { Total intravenous anaesthesia (TIVA) } \\
\text { - } & \text { Isoflurane } \\
\text { - } & \text { Sevoflurane } \\
\text { - } & \text { Miscellaneous (nitrous oxide combinations, desflurane, isoflurane-sevoflurane) }\end{array}$ \\
\hline Local anaesthetic techniques & $\begin{array}{ll}\text { - } & \text { Epidural with local anaesthetic drugs } \\
\text { - } & \text { Epidural without local anaesthetic drugs } \\
\text { - } & \text { Other local techniques } \\
\text { - } & \text { None }\end{array}$ \\
\hline
\end{tabular}

logistic regression to calculate measures of strength of association (crude odds ratios [O.R.s]) for each variable with the presence of regurgitation. Variables with a $P$-value less than 0.3 on univariable regression were considered for inclusion in a multivariable model. The final models were constructed by a manual backwards stepwise procedure where variables with Wald $P$-values of less than 0.05 , or those that changed the estimates of other coefficients in the model by $25 \%$ were retained in the model [22].

Data were analysed using the Minitab statistical software package (Minitab 16.0 for Windows, Minitab Ltd., UK.) Statistical significance was set at $\mathrm{p}<0.05$.

\section{RESULTS}

Data from 5736 general anaesthetics in dogs were reviewed. Seventy five dogs had an episode of regurgitation, giving a prevalence of $1.3 \%$ (95 per cent CI $1.0 \%$ to $1.6 \%)$.

All data underwent univariable logistic regression analysis. Results for categorical and continuous variables are shown in Table 2.

After univariable binomial logistic regression, there was no significant association between the prevalence of regurgitation and the following variables: sex $(p=0.7)$, age of patient $(\mathrm{p}=0.3)$, anaesthetist $(\mathrm{p}=0.7)$, student $(\mathrm{p}=0.9)$, maintenance agent $(\mathrm{p}=0.3)$, local anaesthetic technique used $(p=0.3)$ and length of surgery $(p=0.9)$. 
Table2. Univariable Logistic Regression Model for Categorical and Continuous Variables. The Table Shows the p-Values and the Odds Ratio (OR) with a 95 Percent Confidence Interval (CI)

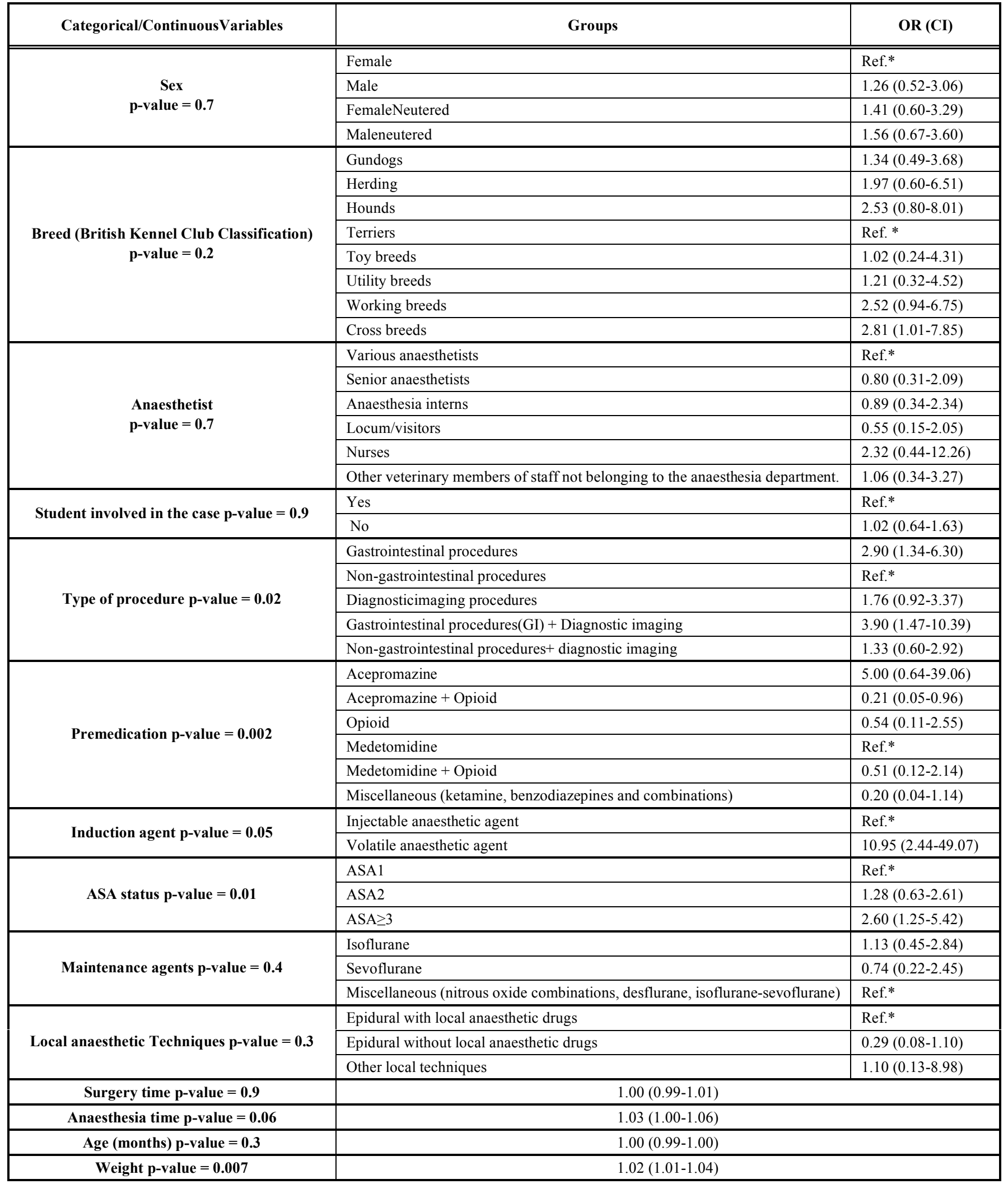

*Referent Variable.

The following variables went forward for inclusion in the multivariable model: breed of patient $(p=0.1)$, ASA status $(p=0.01)$, type of procedure $(p=0.005)$, premedication $(p=$
$0.004)$, induction agents $(p=0.05)$, body weight $(p=0.004)$ and duration of anaesthesia $(\mathrm{p}=0.06)$. 
Multivariable logistic regression analysis identified that dogs undergoing abdominal procedures, imaging procedures and a combination of both imaging and abdominal procedures were at a higher risk of developing regurgitation than dogs undergoing non-abdominal procedures. Furthermore dogs that received acepromazine in combination with an opioid were significantly less likely to suffer from regurgitation than those administered only medetomidine. Dogs classified as an ASA status of 3, 4 and 5 were more likely to experience regurgitation than those with an ASA status of 1. Increase in duration of anaesthesia and greater patient body weight were also associated with the likelihood of an episode of regurgitation occurring (Table 3 ).

Table 3. Multivariable Logistic Regression Model of Factors Associated with Regurgitation in 75 Patients. The Table Shows the $P$ Values and the OR with their 95 Per Cent CI

\begin{tabular}{|l|l|l|}
\hline & \multicolumn{1}{|c|}{ P } & Odds Ratio (CI) \\
\hline \hline Weight & 0.006 & 1.02 \\
& & $(1.01-1.04)$ \\
\hline ASA status & & \\
ASA 1 & Ref.* & Ref.* \\
ASA 2 & 0.37 & $1.39(0.68-2.86)$ \\
ASA>3 & 0.009 & $2.98(1.32-6.76)$ \\
\hline Procedure & & \\
No GI involvement & Ref.* & Ref.* \\
GI involvement & 0.005 & $3.63(1.47-8.95)$ \\
Imaging & 0.006 & $3.13(1.41-6.92)$ \\
GI+Imaging & 0.003 & $5.11(1.73-15.09)$ \\
No GI+Imaging & 0.463 & $1.41(0.57-3.50)$ \\
\hline Premedication & & \\
Medetomidine & Ref.* & Ref.* \\
Acepromazine & 0.38 & $2.59(0.30-22.03)$ \\
Acepromazine+Opioid & 0.023 & $0.17(0.04-0.78)$ \\
Opioid & 0.110 & $0.27(0.05-1.35)$ \\
Miscellaneous & 0.052 & $0.18(0.03-1.01)$ \\
Medetomidine+Opioid & 0.397 & $0.53(0.12-2.30)$ \\
\hline Duration of Anaesthesia(10min intervals) & 0.009 & $1.05(1.01-1.08)$ \\
\hline
\end{tabular}

*Referent Variable.

\section{DISCUSSION}

The prevalence of regurgitation in this clinical population was $1.3 \%$. This is within the range of $0.42 \%-15 \%$ reported in canine experimental population studies $[3,8,10,11]$.

Our results indicated that an increase in anaesthetic duration had a small, but significant, influence on the risk of regurgitation. For example, increasing anaesthesia time by 40 minutes would increase the risk of regurgitation by 1.2 times. Three comparable studies are available, although two of them were primarily interested in GOR and just one of them in regurgitation. Studies looking at the association between age and GOR had mixed results with one of them finding no association between these two variables [8] and a previous one finding a positive association [3]. No association was found between age and regurgitation on a recent study [11].

GOR has been reported to occur, in most cases, shortly after induction of anaesthesia [8]. Although the time at which regurgitation occurred was not recorded in our study, our findings imply that regurgitation can occur after the first 20 minutes of anaesthesia, and indeed is more likely to occur the longer the anaesthetic, although the influence of anaesthetic duration is small. We are unable to provide an explanation for this finding, although the effect of anaesthetic duration still remained significant when confounding influences such as ASA status and different procedures (including the amount of movement/ handling of patient) were accounted for in the statistical analysis.

In this study, no correlation was found between an increase in patient age and the risk of regurgitation, in agreement with Lamata et al. [11]. In contrast, a previous report found a correlation between increase in age and occurrence of GOR [8].

Body mass has been variably reported to be associated with an increased risk of GOR [8] and regurgitation [11]. In the latter study, an increase in body weight was found to be significantly associated with a higher risk of regurgitation. Body condition scores were not recorded for our population and therefore the influence of obesity on regurgitation cannot be reported. In people, obesity has been reported to increase the risk of regurgitation [23]. An alternative explanation may be that larger dogs are more difficult to manoeuvre, needing more than one person to lift and move them, often resulting in being held around the abdomen. The resultant increase in intra-abdominal pressure may predispose such animals to an episode of regurgitation.

In agreement with another study where GOR was investigated, gastrointestinal procedures were found to have a higher risk of regurgitation compared to nongastrointestinal procedures in the present study [8]. An increase in intra-gastric pressure normally induces an increase in lower oesophageal sphincter pressure (LOSP), therefore maintaining the barrier pressure $(\mathrm{BrP})$; but this response is limited, especially in anaesthetised animals and large increases in intra-gastric pressure are likely to induce reflux [24]. Surgical procedures involving the gastrointestinal tract, diagnostic imaging procedures or a combination of both during the same general anaesthetic were also found to increase the risk of regurgitation in this study, especially where multiple imaging procedures were combined during one general anaesthetic. It is possible that a change in depth of anaesthesia (when moving rooms within the diagnostic imaging unit or between this unit and the theatre area), as well as handling the patient and changing its body position may have triggered regurgitation. In those cases that underwent diagnostic imaging procedures and gastrointestinal procedures, the exact time when an episode of regurgitation occurred was not recorded in the data, but diagnostic imaging procedures alone were significantly associated with the incidence of regurgitation and these often require multiple changes in animal position (and may incur anaesthetic depth changes, e.g. bronchoscopy where the endotracheal tube may need to be removed; or increased intra-abdominal pressure e.g. during change of the animal's position or during abdominal ultra sonographic scanning). 
The implication of diagnostic imaging as a causative factor for regurgitation warrants further investigation. These findings areapparently in opposition to those found in a recent study where animals that underwent orthopaedic procedures were at an increased risk of suffering from an episode of regurgitation [11]. This apparent discrepancy may, however, be because imaging procedures were also performed on those orthopaedic cases although this is not clear from their reported results.

ASA status was significantly associated with regurgitation in our study, which is in opposition to previous studies where no association was found [11]. Dogs with ASA status of 3 or higher were at a greater risk of developing intra-anaesthetic regurgitation. Those higher risk patients (e.g. portosystemic shunt, abdominal mass resection, patent ductus arteriosus occlusion and pacemaker placements), may be more unstable under anaesthesia which may partly explain their higher risk of regurgitation.

In our study population, the risk of regurgitation was significantly less in patients that were premedicated with acepromazine in combination with an opioid in comparison to patients administered medetomidine alone. In another study, dogs administered xylazine experienced a 77\% reduction in LOSP (from $47.9 \mathrm{mmHg}$ to $11.7 \mathrm{mmHg}$ ) compared with animals administered acepromazine (62\% reduction, from $47.9 \mathrm{mmHg}$ to $18.6 \mathrm{mmHg}$ ), suggesting that regurgitation might be more likely following the administration of alpha-2-agonists. It was hypothesised that the reduction in LOSP by acepromazine might occur due to inhibition of the effects of 5- hydroxytryptamine, which increases the LOSP in conscious dogs, rather than being due to a primary effect on the central dopaminergic receptors [25]. To the authors' knowledge, the pharmacological mode of action of alpha-2-agonists on the LOSP has not yet been described. A significant difference in $\mathrm{BrP}$ between cats administered acepromazinein comparison to those administered atropine or a combination of drugs was found, where acepromazine alone caused less decrease in $\mathrm{BrP}$ compared to the others [16]. The resting LOSP or $\mathrm{BrP}$ at which animals are at a higher risk of experiencing an episode of regurgitation has not been described in dogs, but in people, a resting LOSP of $13 \mathrm{~cm} \mathrm{H}_{2} \mathrm{O}$ is sufficient to prevent reflux [26]. In contrasts to these studies, other authors have found no association between the administration of acepromazine with the likelihood of an episode of regurgitation occurring [11].

Opioids can delay the emptying of large amounts of gastric content and this may constitute a significant risk factor for regurgitationin patients in emergency situations requiring anaesthesia [24]. Pethidine has been shown to produce phasic contractions of the lower oesophageal sphincter in conscious dogs, with higher minimal pressures compared with atropine, xylazine or acepromazine [25]. Morphine increased the incidence of GOR during the subsequent anaesthetic episode, although no relationship was found between vomiting caused by morphine and the likelihood of experiencing GOR in anaesthetised dogs [7]. In agreement with a previous study [11], we found no association between the use of opioid drugs alone and the incidence of regurgitation, although the different opioids were not separated for analysis in this case.
In our study, we gathered data on individual anaesthetic induction agents/ agent combinations (e.g. propofol, alfaxalone, ketamine with benzodiazepines, and volatile agents) and analysed the data to establish if any individual agent(s)/ combinations were associated with the incidence of regurgitation. No significant associations were found (data not shown) which is in agreement to a previous report [11]. It was only after induction agents were grouped together into injectable agents or inhalation agents that a significant association was found after univariable analysis. After univariable analysis, induction of anaesthesia with volatile agents was a risk factor for regurgitation, but because the inhalation group size was too small $(\mathrm{n}=16)$ compared with the injectable group size $(n=5513)$, this variable could not be included in the final multivariable model. Previous studies comparing the use of propofol and thiopental for induction of anaesthesia in dogs and cats found consistent differences between the effects of these two drugs on the LOSP, although our results suggest that such differences may have little clinical relevance, although thiopental was not commonly used in our study population $[18,27]$.

In this study, the anaesthetic maintenance agent did not affect the risk of regurgitation, which is in accordance with previous studies, where the risk of developing regurgitation or GOR was not significantly affected by the selection of isoflurane, halothane or sevoflurane for maintenance of anaesthesia, respectively [11,19].

\section{CONCLUSION}

Results of the present study suggest that larger dogs, patients with ASA status of 3 or higher, animals undergoing abdominal and/or imaging procedures and animals undergoing a long anaesthetic procedures, are more likely to suffer from an episode of visible regurgitation. Also dogs receiving premedication with medetomidine versus acepromazine with an opioid are at a higher risk of regurgitation.

In order to elucidate risk factors in more depth, further information is needed from multi-centre studies. Additionally, follow-up studies could be of use in order to determine outcomes in patients reported to have regurgitated under anaesthesia.

Knowing these risk factors should inform clinicians about high risk cases so they are vigilant and, where possible, can manage modifiable risks, such as type of premedication or minimising the length of anaesthesia as much as possible, as well as being aware of the procedures or patients at a higher risk of suffering from regurgitation so faster action can be elicited.

\section{CONFLICT OF INTEREST STATEMENT}

None of the authors has any financial or personal relationships that could inappropriately influence or bias the content of the paper.

\section{ACKNOWLEDGEMENTS}

The authors would like to thank their colleagues of the Small Animal Teaching Hospital that anaesthetised, cared 
for and made comprehensive records which allowed this study to be undertaken.

\section{REFERENCES}

[1] Galatos AD, Rallis T, Raptopoulos D. Post anaesthetic oesophageal stricture formation in three cats. J Small Anim Pract 1994; 35: 63842 .

[2] Sondheimer JM. Continuous monitoring of distal esophageal pH: A diagnostic test for gastroesophageal reflux in infants. J Pediatr 1980; 96: 804-7.

[3] Wilson DV, Evans AT, Miller RA. Effects of preanesthetic administration of morphine on gastroesophageal reflux and regurgitation during anesthesia in dogs. Am J Vet Res 2005; 66: 386-90.

[4] Hashim MA, Waterman AE. Determination of the length and position of the lower oesophageal sphincter (LOS) by correlation of external measurements with combined radiographic and manometric estimations in the cat. Brit Vet J 1992; 148: 435-44.

[5] Mendelson CL. Aspiration of stomach contents into lungs during obstetric anesthesia. Am J Obstet Gynecol 1946; 52: 129-31.

[6] Pearson H, Darke PGG, Gibbs C, et al. Reflux oesophagitis and stricture formation after anaesthesia: a review of seven cases in dogs and cats. J Small Anim Pract 1978; 19: 507-19.

[7] Wilson DV, Walshaw R. Post anaesthetic oesophageal dysfunction in 13 dogs. J Am Anim Hosp Assoc 2004; 40: 455-60.

[8] Galatos AD, Raptopoulos D. Gastro-oesophageal reflux during anaesthesia in the dog: effect of age, positioning and type of surgical procedures. Vet Rec 1995a; 137: 513-6.

[9] Galatos AD, Raptopoulos D. Gastro-oesophageal reflux during anaesthesia in the dog: effect of preoperative fasting and premedication. Vet Rec 1995b; 137: 479-83.

[10] Wilson DV, Evans AT, Mauer WA. Infuence of metoclopramide on gastroesophageal reflux in anesthetized dogs. Am J Vet Res 2006a; 67: 26-31

[11] Lamata $\mathrm{C}$, Loughton $\mathrm{V}$, Jones $\mathrm{M}$, et al. The risk of passive regurgitation during general anaesthesia in a population of referred dogs in the UK. Vet Anaesth Analg 2012; 39: 266-74.

[12] Blitt CD, Gutman HL, Cohen DD, et al. "Silent" regurgitation and aspiration during general anesthesia. Vet Anesth Analg 1970; 49: 707-13.

[13] Benington S, Severn A. Preventing aspiration and regurgitation. Anaesth Intens Care Med 2007; 8: 368-72.
[14] Savvas I, Rallis T, Raptopoulos D. The effect of pre-anaesthetic fasting time and type of food on gastric content volume and acidity in dogs. Vet Anesth Analg 2009; 36: 539-46.

[15] Cotton BR, Smith G. The lower oesophageal sphincter and anaesthesia. Br J Anaesth 1984; 56: 37-46.

[16] Hashim MA, Waterman AE. Effects of acepromazine, pethidine and atropine premedication on lower oesophageal sphincter pressure and barrier pressure in anaesthetised cats. Vet Rec 1993; 133: $158-60$.

[17] Panti A, Bennett RC, Corletto F, et al. The effect of omeprazole on oesophageal $\mathrm{pH}$ in dogs during anaesthesia. J Small Anim Pract 2009; 50: 540-4

[18] Raptopoulos D, Galatos AD. Gastro-oesophageal reflux during anaesthesia induced with either thiopentone or propofol in the dog. J Vet Anaesth 1997; 24: 20-2.

[19] Wilson DV, Boruta DT, Evans AT. Influence of halothane, isoflurane and sevoflurane on gastroesophageal reflux during anesthesia in dogs. J Am Vet Res 2006b; 67: 1821-5.

[20] Waterman AE, Hashim MA, Pearson H. Effect of body position on esophageal and gastric pressures in the anaesthetised dog. J Small Anim Pract 1995; 36: 196-200.

[21] Anagnostou TL, Savvas I, Kazakos, GM, et al. Effect of endogenous progesterone and oestradiol-17beta on the incidence of gastro-oesophageal reflux and on the barrier pressure during general anaesthesia in the female dog. Vet Anaesth Analg 2009; 36: 308-18

[22] Hosmer DW, Lemeshow S. Model-building strategies and methods for logistic regression. Applied Logistic Regression $2^{\text {nd }}$ ed. New York: John Wiley \& Sons, Inc. 2000.

[23] Friedenberg FK, Xanthopoulos M, Foster GD, et al. The association between gastroesophageal reflux disease and obesity. Am J Gastroenterol 2008; 103: 2111-22.

[24] Hardy JF. Large volume gastro-esophageal reflux: a rationale for risk reduction in the preoperative period. Can J Anaest 1988; 35: 162-73.

[25] Strombeck D, Harrold D. Effects of atropine, acepromazine, meperidine, and xylazine on gastroesophageal sphincter pressure in the dog. J Vet Res 1985; 46: 963-5.

[26] Haddad JK. Relation of gastroesophageal reflux to yield sphincter pressures. Gastroenterol 1970; 58: 175-84

[27] Galatos AD, Savvas I, Prassinos NN, et al. Gastro-oesophageal reflux during thiopentone or propofol anaesthesia in the cat. J Vet Med 2001; 48: 287-94.

This is an open access article licensed under the terms of the Creative Commons Attribution Non-Commercial License (http: //creativecommons.org/licenses/bync/3.0/) which permits unrestricted, non-commercial use, distribution and reproduction in any medium, provided the work is properly cited. 